\title{
Factores de riesgo de mortalidad en pacientes que presentaron peritonitis en diálisis peritoneal crónica en el Hospital Militar Central de Bogotá
}

\author{
John Alejandro Camargo ${ }^{1}$, Gustavo Quiroz ${ }^{1}$, Paquita Méndez², M Castañeda ${ }^{2}$, \\ Juan Guillermo Vargas ${ }^{1,2}$, Roberto D Achiardi ${ }^{2}$, Jorge Enrique Echeverri ${ }^{1,2}$ \\ 1 Universidad Militar Nueva Granada, Bogotá, Colombia, \\ 2 Servicio de nefrología RTS - Hospital Militar Central, Bogotá, Colombia
}

\begin{abstract}
Resumen
La diálisis peritoneal crónica (DPC) es una de las opciones más frecuentes de inicio de terapia de remplazo renal crónico en Colombia. La presencia de complicaciones inherentes a la terapia tiene baja incidencia, sin embargo, la peritonitis asociada con DPC se ha relacionado con el posterior cambio de modalidad terapéutica a hemodiálisis crónica y con aumento de las tasas de hospitalización. Se desarrolló un estudio de casos y controles con el fin de evaluar la mortalidad y posibles factores de riesgo asociados en los pacientes que presentaron peritonitis en DPC en el periodo comprendido entre el 1 de enero de 2010 y el 31 de diciembre de 2012. Los factores de riesgo identificados para desarrollo de peritonitis y mortalidad no tuvieron significancia estadística. Los casos de peritonitis atribuidos a fatiga del cuidador, presentaron mayor riesgo de recurrencia y recaída de la peritonitis. En cuanto al riesgo de mortalidad se encontró que la probabilidad para la presentación de dicho desenlace fue 1,75 veces mayor en los hombres, 2.2 veces mayor en los pacientes mayores de 75 años, 3.5 veces mayor en los diabéticos y 5.6 veces más elevado en los pacientes hipertensos.
\end{abstract}

Palabras clave: Diálisis peritoneal crónica, peritonitis, mortalidad.

\section{Risk factors for mortality in patients who developed peritonitis in chronic peritoneal dialysis in the Hospital Militar Central de Bogotá}

\begin{abstract}
In Colombia, chronic peritoneal dialysis (CPD) is one of the most common forms of onset of chronic renal replacement therapy. Complications associated with therapy are low, however, peritonitis associated with CPD has been associated with subsequent change of treatment modality to chronic hemodialysis and with increased rates of hospitalization. We performed a control and case study in order to assess the mortality associated risk factors in patients who developed peritonitis in CPD between january 1, 2010 and december 31 , 2012. Risk factors identified for development of peritonitis and mortality were not statistically significant. Cases of peritonitis attributed to caregiver fatigue had a higher risk of recurrence and relapse of peritonitis. Regarding the risk of mortality, we found that the probability for this outcome was 1.75 times higher in men, 1.6 times higer in male, 2.2 times higher in patients older than 75 years, 3.5 times higher in diabetics and 5.6 times higher in hypertensive patients.
\end{abstract}

Key words: chronic peritoneal dialysis, peritonitis, mortality. 


\section{Introducción}

L os factores de riesgo para presentar peritonitis asociada con DPC han sido evaluados en múltiples estudios ${ }^{1-6}$, en los cuales se suelen contemplar 2 escenarios para su interpretación: los factores de riesgo que se relacionan con el primer episodio de peritonitis y los relacionados con las recurrencias; teniendo en cuenta que son las peritonitis recurrentes las que se han relacionado con mayor frecuencia de disfunción de membrana peritoneal, lo cual puede llevar a cambio de modalidad de tratamiento.

En un estudio que utiliza la base de datos USRDS, de 11.975 pacientes prevalentes con DPC entre 1994 y 1997, los principales factores de riesgo identificados fueron edad menor de 44 años, diabetes mellitus, raza negra e insuficiencia cardíaca congestiva ${ }^{3}$. En el registro ANZDATA en Australia y Nueva Zelanda, entre 3.162 pacientes incidentes con DPC tratados entre 1999 y 2003 se identificaron como factores de riesgo para peritonitis la obesidad, la raza aborigen australiana y la edad avanzada ${ }^{4}$. En Canadá, en el registro POET, entre 4.247 pacientes incidentes con DPC entre 1996 y 2005, los principales factores de riesgo identificados fueron la transferencia de hemodiálisis crónica a DPC, la diabetes mellitus y entre los diabéticos, pertenecer al género femenino ${ }^{5}$.

En el estudio BRAZPD en Brasil, entre 2.032 pacientes incidentes con DPC entre 2004 y 2007, se encontró que el bajo nivel educativo, residir en el norte del país, vivir a una distancia mayor de $50 \mathrm{~km}$ del centro de diálisis y pertenecer a un centro de diálisis con más de 150 pacientes, fueron factores de riesgo independientes para peritonitis de $\mathrm{DPC}^{6}$.

Se han identificado algunos factores de riesgo para el desarrollo de peritonitis asociada con DPC; sin embargo, los factores de riesgo de mortalidad en este grupo poblacional aún son desconocidos, en especial en la población con recurrencia.

\section{Objetivos e hipótesis}

Evaluar los factores de riesgo de mortalidad en pacientes de diálisis crónica que presentaron perito- nitis asociada con diálisis peritoneal crónica, en el Servicio de Nefrología del Hospital Militar Central de Bogotá, en el período comprendido entre el 1 de enero de 2010 y el 31 de diciembre de 2012.

\section{Diseño}

Estudio observacional de casos y controles anidado en una cohorte.

\section{Materiales y métodos}

Estudio retrospectivo de casos y controles, al cual ingresaron pacientes mayores de 18 años de edad, con diagnóstico confirmado de enfermedad renal crónica estadio 5 de acuerdo con las Guías KDIGO de 2012 y diagnóstico confirmado de peritonitis asociada con diálisis peritoneal crónica, de acuerdo con la Guía de Infecciones Asociadas a Diálisis Peritoneal de $2010^{7,8}$.

Para la escogencia de los casos se incluyeron los pacientes del programa de DPC del Hospital Militar Central de Bogotá, Colombia, que presentaron peritonitis y fallecieron durante el periodo de seguimiento propuesto. Para los controles se incluyeron aquellos pacientes con peritonitis asociada con DPC que no fallecieron, registrando las características demográficas, clínicas o paraclínicas durante el periodo de seguimiento.

El análisis estadístico descriptivo incluye, para las variables continuas, el cálculo de medidas de tendencia central y de dispersión tales como promedios y desviaciones estándar o medianas para las variables numéricas, y frecuencias absolutas y porcentajes para las variables categóricas.

Adicionalmente, se efectuó un análisis bivariado encaminado a identificar los factores de riesgo para mortalidad entre los pacientes reclutados. Para los datos numéricos se calculó la diferencia de medias entre los grupos utilizando t- student (cuando la distribución era normal) o la diferencia de la media de rangos entre los grupos empleando la prueba de Wilcoxon (cuando la distribución no era normal). Para las variables cualitativas nominales se identificaron las posibles asociaciones por medio $\chi^{2}$ (chi cuadrado) para variables categóricas. 
Finalmente, se calculó el OR con su respectivo intervalo de confianza en aquellas variables que mostraron alguna asociación. El nivel de significancia estadístico fue definido como una $\mathrm{p}<0.05$. Todos los análisis estadísticos se realizaron por medio del paquete estadístico SPSS.

\section{Resultados}

Durante el período comprendido entre enero 1 de 2010 y diciembre 31 de 2012 se presentaron un total de 64 episodios de peritonitis en pacientes en DPC. Se analizaron un total de 127 pacientes manejados con DPC, 62 que cursaron con peritonitis y 65 que no presentaron esa complicación. No se encontraron diferencias estadísticamente significativas con respecto a las características demográficas y clínicas entre los 2 grupos. En ambos casos predominaron los pacientes de género masculino (71\% y $69.2 \%$ respectivamente) y los mayores de 55 años (59.7\% vs. $63.1 \%$ respectivamente). Por otra parte, la modalidad de diálisis más frecuentemente utilizada fue la diálisis peritoneal automatizada (APD) (59.7\% y $72.3 \%$ respectivamente) y las comorbilidades más observadas fueron la diabetes mellitus $(35.4 \%)$ y la hipertensión arterial (75.3\%).

Entre los pacientes con peritonitis de DPC, los hallazgos clínicos que se evidenciaron con mayor frecuencia fueron la presencia de líquido turbio (98.4\%) y de dolor abdominal (83.9\%), seguidos por taquicardia (35.5\%) y vómito (32.3\%) (Figura 1).

Al evaluar las características del líquido peritoneal se encontró que el recuento de leucocitos osciló entre 40 y 60940 , con un promedio de $6369,7 \pm 11102$ y un porcentaje de neutrófilos entre 64 y $100 \%$ con promedio de $89.07 \pm 8.93$.

Con respecto a la microbiología el $62.9 \%$ de los estudios de Gram fueron reportados como negativos. Entre aquellos en los que se identificó algún germen, los cocos Gram positivos fueron los más frecuentes $(24.2 \%)$, seguidos por bacilos Gram negativos (8.1\%) y por cocos Gram negativos (1.6\%). En la tabla 1 se describen los microorganismos que fueron aislados en los cultivos: en el $70.9 \%$ de los casos se aisló algún germen $(n=44)$.
Con respecto a los factores de riesgo para desarrollar peritonitis asociada con DPC; se evidenció que los pacientes de género masculino tuvieron mayor probabilidad para dicho desenlace (OR 1.086 IC95\% 0.508 - 2.324). De manera similar, se encontró que los pacientes con antecedente de diabetes mellitus (OR 1.152 IC 95\% 0.552 - 2.404), de hipertensión arterial (OR 1.852 IC95\% 0.552 - 2.404), de glomerulonefritis (OR 2.590 IC 95\% 0.754 - 8.895), de lupus eritematoso sistémico (OR 1.052 IC 95\% 0.251 - 4.403) o de enfermedad autoinmune (OR 1.252 IC 95\% 0.396 - 3.955), presentaron incremento de riesgo para peritonitis asociada con DPC.

Con respecto a los factores de riesgo para recurrencia, recidiva o recaída de la peritonitis asociada con DPC, se evidenció que la probabilidad de este desenlace fue mayor entre los pacientes de género masculino (OR 1.667 IC 95\% 0.405 - 6.861), manejados bajo la modalidad de APD (OR 1.994 IC 95\% 0.534 - 7.079), con antecedente de glomerulonefritis (OR 3.44 IC $95 \% 0.780$ - 15.172) o de enfermedad autoinmune (OR 1.433 IC 95\% 0.247 - 8.333), cuyo episodio inicial de peritonitis fue secundario a falla de la técnica de conexión (OR 1.667 IC 95\% 0.405 -6.861) o asociada al cuidador (OR 3 IC $95 \% 0.873$ $-10.312)$.

En cuanto al riesgo de mortalidad se encontró que la probabilidad para la presentación de dicho desenlace fue 1.7 veces mayor entre los pacientes masculinos, 2.2 entre los pacientes con edad mayor de 75 años, 3.5 entre los pacientes diabéticos y 5.6 veces más elevada entre los pacientes hipertensos.

\section{Discusión}

La peritonitis asociada con DPC es una complicación frecuente de los pacientes que reciben esta modalidad de diálisis crónica. La evaluación del líquido peritoneal constituye un punto esencial en el diagnóstico de peritonitis, siendo la turbidez del líquido peritoneal el principal signo clínico. Los procesos infecciosos peritoneales repetitivos llevan a falla de la membrana y constituyen un marcador de calidad dentro de los programas de las unidades renales, tomando un papel primordial los procesos de prevención más que de intervención luego de los eventos. 
La peritonitis asociada con DPC ha sido reportada como de baja mortalidad, sin embargo, es una causa frecuente de deterioro de la membrana peritoneall. La peritonitis asociada con DPC constituye la principal causa para cambio de terapia de remplazo renal crónico y de disfunción de la membrana peritoneal a largo plazo8. Los esfuerzos para el control de la peritonitis en DPC, van dirigidos hacia la identificación de los factores de riesgo para desarrollo de los procesos infecciosos intraperitoneales, como son la identificación y manejo de infecciones del orificio de salida, infección del túnel y otros factores de riesgo como son diabetes, obesidad y estreñimiento9. El avance en las técnicas de implantación de los catéteres y el retiro oportuno cuando no hay respuesta al tratamiento (5 días), recaída temprana (S. epidermidis) o en los casos de infecciones por gérmenes multirresistentes (pseudomonas, hongos, BLES), ha disminuido la tasa de pérdidas funcionales del peritoneo y se ha relacionado con menor cambio de modalidad de terapia ${ }^{10,11}$.

El presente estudio hizo una evaluación cercana de los posibles factores asociados con la aparición de peritonitis de DPC, encontrando que, factores asociados con la técnica de conexión y la participación del cuidador en el manejo del paciente en DPC, pueden relacionarse con la presentación de estos eventos infecciosos, siendo importante el seguimiento y reentrenamiento de ellos. De igual forma consideramos importante evaluar periódicamente elementos emocionales, adherencia y compromiso de quienes participan en la realización de la terapia en casa, con el fin de poder mejorar la oportunidad de las intervenciones $^{12-14}$.

La mortalidad en el grupo poblacional en diálisis peritoneal crónica ha sido reportada entre 10 a $20 \%$ anual, siendo las infecciones parte de las condiciones encontradas. La tasa de mortalidad de las peritonitis difiere entre los diversos reportes de la literatura, encontrándose entre el 5 y el $30 \%{ }^{15,16}$. Es así como, acorde con reportes Australianos, cerca del $20 \%$ de los pacientes que fallecen en DPC pueden tener un episodio de peritonitis en los 30 días previos, con un riesgo 6 veces mayor de tener un episodio infeccioso reciente al comparar el periodo de 30 días con el de 6 meses previos a la muerte ${ }^{17,18}$. Sin embargo, es difícil definir los factores de riesgo, las condiciones asociadas a estos desenlaces fatales y el tiempo en el cual el episodio de peritonitis pudiera estar relacionado con la mortalidad ${ }^{19,20}$. Hasta la actualidad los estudios que han analizado esta relación no pueden ser interpretados como evidencia de causalidad ${ }^{18,21}$.

Se identificaron factores de riesgo para mortalidad en la población con antecedente de peritonitis de DPC (edad, diabetes mellitus e hipertensión arterial), similar a los reportados en la población general en diálisis crónica. Dentro de la base poblacional estudiada la mortalidad general se presentó luego de 30 días de diagnóstico del proceso infeccioso peritoneal, lo cual podría explicar los resultados encontrados. Es importante seguir indagando en estudios prospectivos, que puedan caracterizar la población en diálisis peritoneal crónica que tenga mayor riesgo de tener un desenlace fatal asociado con la peritonitis.

\section{Conclusiones}

El presente estudio tuvo como propósito hacer una evaluación cercana de los posibles factores asociados a mortalidad, sin embargo, no encontramos significancia estadística en las variables evaluadas lo cual no permite generar conclusiones definitivas. Los factores de riesgo para mortalidad (edad, diabetes mellitus e hipertensión arterial) identificados en la población con peritonitis de DPC, no difieren de los factores reportados en la literatura para pacientes en diálisis peritoneal crónica.

\section{Conflicto de interés}

Los autores declaran no tener ningún conflicto de interés. 


\section{Figura 1}

Hallazgos clínicos de los pacientes con peritonitis de diálisis peritoneal crónica

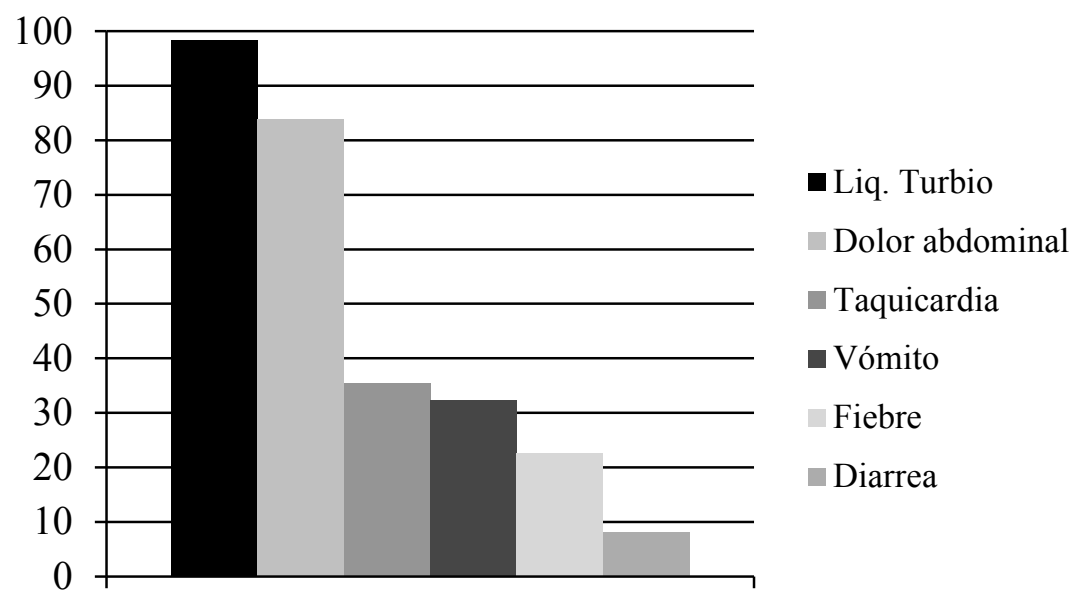

Categoría 1

Tabla 1

Agente etiológico de peritonitis asociada con diálisis peritoneal crónica

\begin{tabular}{|l|l|l|}
\multicolumn{1}{|c|}{ Organismo } & $\mathrm{n}$ & $\mathbf{4}$ \\
\hline Gram positivos & $\mathbf{2 6}$ & $\mathbf{4 1 , 9}$ \\
\hline Staphylococcus epidermis & 9 & 12,9 \\
\hline Staphylococcus aureus & 8 & 4,8 \\
\hline Enterococo fecalis & 3 & 3,2 \\
\hline Streptococcus intermedius & 2 & 1,6 \\
\hline Staphylococcus hominis & 1 & 1,6 \\
\hline Staphylococcus warneri & 1 & 1,6 \\
\hline Staphylococcus anginosus & 1 & $\mathbf{2 7 , 4}$ \\
\hline Gram negativos & $\mathbf{1 7}$ & 8,1 \\
\hline Klebsiella pneumoniae & 5 & 6,4 \\
\hline Escherichia coli & 4 & 3,2 \\
\hline Enterobacter cloacae & 2 & 3,2 \\
\hline Streptococcus marcescens & 2 & 1,6 \\
\hline Haemophylus spp. & 1 & 1,6 \\
\hline Klebsiella oxytoca & 1 & 1,6 \\
\hline Pseudomona aeruginosa & 1 & 1,6 \\
\hline Stenotrophomona maltophilia & 1 & $\mathbf{1 , 6}$ \\
\hline Hongos & $\mathbf{1}$ & 1,6 \\
\hline Candida albicans & 1 & $\mathbf{2 7 , 4}$ \\
\hline Cultivo negativo & $\mathbf{1 7}$ & $\mathbf{1 , 6}$ \\
\hline Sin dato & $\mathbf{1}$ & \\
\hline
\end{tabular}




\section{Bibliografía}

1. Davenport A. Peritonitis remains the major clinical complication of peritoneal dialysis: the London, UK, peritonitis audit 2002-2003. Perit Dial Int. 2009;29(3):297-302.

2. Nieto-Ríos JF, Díaz-Betancur JS, Arbeláez-Gómez M, et al. Peritoneal dialysis-related peritonitis: twenty-seven years of experience in a Colombian medical center. Nefrología. 2014;34(1):88-95.

3. Oo TN, Roberts TL, Collins AJ: A comparison of peritonitis rates from the United States Renal Data System database: CAPD versus continuous cycling peritoneal dialysis patients. Am J Kidney Dis 2005;45:372-380.

4. Lim WH, Johnson DW, McDonald SP: Higher rate and earlier peritonitis in Aboriginal patients compared to non-Aboriginal patients with end-stage renal failure maintained on peritoneal dialysis in Australia: Analysis of ANZDATA. Nephrology (Carlton) 2005;10:192-197.

5. Nessim SJ, Bargman JM, Austin PC, et al. Predictors of peritonitis in patients on peritoneal dialysis: results of a large, prospective Canadian database. Clin J Am SocNephrol. 2009 Jul;4(7):1195-1200.

6. Martín LC, Caramori JC, Fernández N, et al. Brazilian Peritoneal Dialysis Multicenter Study BRAZPD Group. Geographic and educational factors and risk of the first peritonitis episode in Brazilian Peritoneal Dialysis study (BRAZPD) patients. Clin J Am Soc Nephrol. 2011;6(8):1944-1951.

7. Li PK, Szeto CC, Piraino B, et al. International Society for Peritoneal Dialysis. Peritoneal dialysis-related infections recommendations: 2010 update. Perit Dial Int. 2010 Jul-Aug;30(4):393-423.

8. Stevens PE, Levin A; Kidney Disease: Improving Global Outcomes Chronic Kidney Disease Guideline Development Work Group Members. Evaluation and management of chronic kidney disease: synopsis of the kidney disease: improving global outcomes 2012 clinical practice guideline. Ann Intern Med. 2013;158(11):825-830.

9. Piraino B, Bailie GR, Bernardini J, et al. Peritoneal Dialysis-Related Infections. Recommendations 2005 UPDATE. Perit Dial Int 2005;25:107-131.

10. Li PK, Law MC, Chow KM, et al. Comparison of clinical outcome and ease of handling in two double-bag systems in continuous ambulatory peritoneal dialysis: a prospective, randomized, controlled, multicenter study. Am J Kidney Dis 2002;40(2):373-380.

11. Strippoli GFM, Tong A, Johnson D, et al. Catheter-related interventions to prevent peritonitis in peritoneal dialysis: a systematic review of randomized controlled trials. J Am SocNephrol 2004;15(10):2735-2746.

12. Troidle L, Watnick S, Wuerth $\mathrm{D}$, et al. Depression and its association with peritonitis in long-term peritoneal dialysis patients Am J Kidney Dis 2003;42:350-354.

13. Jager KJ, Merkus MP, Dekker FW, et al. NECOSAD Study Group: Mortality and technique failure in patients starting chronic peritoneal dialysis: Results of The Netherlands Cooperative Study on the Adequacy of Dialysis. Kidney Int 1999;55:1476-1485.

14. Johansen KL, Chertow GM, Jin C, Kutner NG: Significance of frailty among dialysis patients. J Am SocNephrol 2007;18:2960-2967.

15. Fang W, Qian J, Lin A, et al. Comparison of peritoneal dialysis practice patterns and outcomes between a Canadian and a Chinese Centre. Nephrol Dial Transplant 2008;23:4021-4028.

16. Hiramatsu M; Japanese Society for Elderly Patients on Peritoneal Dialysis: How to improve survival in geriatric peritoneal dialysis patients. Perit Dial Int 2007;27(S2):S185-S189.

17. Excell L, Livingston B, McDonald SP: ANZDATA Registry Report 2010, Adelaide, South Australia, Australia and New Zealand Dialysis and Transplant Registry, 2010.

18. Boudville N, Kemp A, Clayton P, et al. Recent peritonitis associates with mortality among patients treated with peritoneal dialysis. J Am SocNephrol 2012;23(8):1398-1405.

19. Sipahioglu MH, Aybal A, Unal A, et al. Patient and technique survival and factors affecting mortality on peritoneal dialysis in Turkey: 12 years' experience in a single center. Perit Dial Int 2008;28:238-245.

20. Szeto CC, Kwan BC, Chow KM, et al. Repeat peritonitis in peritoneal dialysis: Retrospective review of 181 consecutive cases. Clin J Am Soc Nephrol 2011;6:827-833.

21. Pérez Fontan M, Rodríguez-Carmona A, García-Naveiro R, et al. Peritonitis-related mortality in patients undergoing chronic peritoneal dialysis. Perit Dial Int 2005;25:274-284. 